\title{
¿CÓMO SON LAS MUJERES QUE LIDERAN PYMES? UN ESTUDIO APLICADO EN COSTA RICA
}

\author{
What are female SMEs leaders like? Applied study in Costa Rica
}

\author{
Marcela Rodríguez-Jiménez \\ Bachiller Universitario, investigadora Escuela de Administración de Empresas, \\ Instituto Tecnológico de Costa Rica. Cartago- Costa Rica, marce.rod0387@gmail.com
}

Juan Carlos Leiva

PhD en Dirección de Empresas, profesor catedrático, Escuela de Administración de Empresas, Instituto Tecnológico de Costa Rica. Cartago- Costa Rica, jleiva@itcr.ac.cr

\section{Carmen Castrejón-Mata}

PhD en Administración, profesora investigadora, Universidad de Guanajuato, Guanajuato - México, carsais@hotmail.com

\section{Cómo citar / How to cite}

Rodriguez-Jimenez, M., Leiva, J. y Castrejón-Mata, Carmen. (2017). ¿Cómo son las mujeres que lideran PYMES? Un estudio aplicado en Costa Rica. Revista CEA, 3(5), 29-40.

Recibido: 25 de agosto de 2016

Aceptado: 28 de octubre de2016

\section{Resumen}

El estudio analiza las características de las emprendedoras costarricenses y sus empresas, buscando aportar conocimiento, así como propuestas de apoyo para ese conglomerado. La metodología combina un enfoque cuantitativo a partir de datos del segundo informe nacional de las Pymes del Observatorio de Costa Rica en la materia, así como cualitativo mediante entrevistas a informantes claves. Los resultados muestran cómo las mujeres inician un negocio motivadas principalmente por independencia, en edades entre los 35 y 49 años, su nivel de escolaridad al iniciar es universitario mayormente, desarrollan negocios principalmente en el sector servicios y su participación en las micros, pequeñas y medianas empresas formales del país es de $16.98 \%$. Los principales obstáculos percibidos: su liderazgo y empoderamiento, así como los roles en la sociedad. Como recomendaciones, se plantea implementar programas educativos fomentadores del empoderamiento y programas de acompañamiento con desarrollo de negocios.

Palabras clave: emprendedores, Pymes, mujeres, Costa Rica, espíritu emprendedor femenino.

\begin{abstract}
This study is an analysis of the characteristics of Costar Rican female entrepreneurs and their businesses. The aim is to provide insight and make proposals to support this economic sector. The method involves a quantitative approach based on the data in the second
\end{abstract}


national report by the Observatory of Costa Rican SMEs. It also includes a qualitative approach: Key Informant Interviews. The results show that women start businesses between 35 and 49 years of age, mainly motivated by the idea of independence. Most of them have higher educational level and most of their businesses are conducted in the service sector. Their market share in the formal SMEs in the country is $16.98 \%$. The main challenges they face are leadership and empowerment, as well as their roles in society. The authors recommend the implementation of educational programs that promote empowerment and businesses development support programs.

Keywords: entrepreneurs, SMEs, women, Costa Rica, female entrepreneurial spirit.

\section{INTRODUCCIÓN}

La fórmula del éxito es estudiar mucho, encontrar una buena pareja y trabajar duro. Esta frase era una consigna aceptada en las décadas de 1960 y 1970 en la mayoría de nuestras sociedades occidentales. A partir de los años 80 eso fue cambiando. Las grandes compañías iniciaron su expansión, pero aun así no fueron capaces de proporcionar trabajo a todas las personas que lo necesitaban. Por su lado, los gobiernos tampoco pudieron crear condiciones económicas que generaran suficientes fuentes de empleo. Dado lo anterior, surge el interés por el emprendimiento, como opción de vida y fuente de empleo para muchas personas (Olmos y Arrayales, 2007).

Concretamente, por medio del emprendimiento, se pueden obtener beneficios tanto para el país en cuanto a la generación de empleos, la estimulación de la economía, así como para las personas porque se genera autoempleo, se promueve la autorrealización que conlleva para muchas de ellas poseer su propia empresa y el cumplimiento de sus sueños.

Lo anteriormente señalado no hace distingos de género, aplica tanto a hombres como mujeres. Por ello el estudio del proceso emprendedor se ha efectuado mayoritariamente sin distinción de género, pero es indudable que el entorno de hombres y mujeres no siempre es igual para emprender, lo cual ha producido que se conozca poco sobre el proceso emprendedor femenino en concreto (GEM, 2007).

Algunos trabajos han intentado lanzar alguna luz sobre dicho proceso emprendedor femenino; en Latinoamérica el Women's Report, Global Entrepreneurship Monitor (GEM, 2012), expone resultados del proceso emprendedor para diversos países. Asimismo, se tiene una herramienta creada por el Fondo Multilateral de Inversiones (FOMIN), miembro del Banco Interamericano de Desarrollo, llamada Women's Entrepreneurial Venture Scope (WEVentureScope, 2013), el cual propone un índice (desarrollado en alianza con The Economist Intelligence Unit) calificador del ecosistema emprendedor para las mujeres de América Latina y el Caribe. Estos valiosos esfuerzos constituyen una base general de información, incluyendo varias naciones entre las cuales está Costa Rica, pero persiste la necesidad de ampliar el conocimiento de este tema en el país (Brenes y Bermúdez, 2013).

Dado lo anterior, nuestro objetivo es analizar cómo se caracterizan las emprendedoras costarricenses propietarias de pequeñas y medianas empresas (Pymes), considerando aspectos específicos como las motivaciones para iniciar su negocio, las características personales y de sus empresas, así como los principales obstáculos y apoyos percibidos por ellas. 
Lo que resta del trabajo ha sido ordenado así. En primera instancia una sección describiendo lo que se conoce del emprendimiento femenino en Costa Rica, proseguido por la metodología del estudio, los resultados y se cierra con las conclusiones.

\section{EL EMPRENDIMIENTO FEMENINO EN COSTA RICA}

El estudio del emprendimiento puede abordarse desde diversas perspectivas. Una de las más aceptadas es enfocar la atención en dos aspectos: quiénes son las personas emprendedoras y cómo es el entorno en el cual se desenvuelven (Kantis, Angelelli y Koening, 2004). En este trabajo, acorde a los objetivos planteados, el primer aspecto lo conceptualizamos como las motivaciones y características de las personas emprendedoras; y el segundo, como los obstáculos y apoyos percibidos.

En cuanto al primero aspecto, el Global Entrepreneurship Monitor (GEM) de Costa Rica (Lebendiker, Petry, Herrera y Velázquez, 2012), evalúa la situación del emprendimiento en nuestro país, mencionando que el $78 \%$ de los emprendedores en etapas iniciales se encuentra motivado por oportunidad (34.6\% buscaba incrementar los ingresos y $13.8 \%$ independencia económica), el $27.7 \%$ tiene motivos mixtos y el $21 \%$ está motivado por necesidad, es decir, porque no tienen otra forma de ganarse la vida.

En cuanto a las características, es bastante aceptado identificar a la persona emprendedora por su necesidad de logro. Pero la lista de rasgos incluye, además, otros como el deseo de ser independiente, la capacidad de tolerar la ambigüedad y el riesgo, la perseverancia, así como la autoconfianza (Kantis, et al., 2004 citando a McClelland, 1961). Unido a lo anterior, también se distingue por su capacidad de aprender, lo que le permite a pesar de los fracasos y frustraciones, lograr sus objetivos quebrando patrones y creando su propio nuevo orden (Gilder, 1984).

No obstante, en el caso concreto costarricense no se sabe mucho sobre las mujeres emprendedoras y sus empresas. En uno de los pocos trabajos al respecto, Brenes y Bermúdez (2013) señalan la existencia de diferencias significativas en cuanto al tamaño de las empresas, el conocimiento, así como la experiencia de las mujeres respecto de los hombres en el sector empresarial. Además, las mismas autoras identifican en las mujeres mayores limitaciones para emprender de manera individual y más dependencia de los ingresos generados por sus negocios. Según indican, estas diferencias por género se traducen en una brecha de ingresos con desigualdad de oportunidades donde las mujeres ven limitado tanto el acceso, como su participación en el ámbito empresarial costarricense.

Ligando lo anterior con el entorno en el cual se desenvuelven las mujeres emprendedoras, segundo aspecto de interés en el presente artículo, según la Organización de las Naciones Unidas (2010) las mujeres de América Latina y el Caribe enfrentan barreras mucho más complejas en la participación de la economía formal que sus contrapartes en países desarrollados. Aspectos como las escasas políticas públicas, inadecuados programas de estímulo a la actividad emprendedora, exceso de normativas y falta de acceso al crédito son los más destacables. Por ello, dicho informe recomienda a la hora de diseñar programas de apoyo, tener en cuenta las realidades contextuales referidas a factores macroeconómicos, condiciones institucionales, de infraestructura, cuestiones normativas, crediticias y financieras. 
En el caso de Costa Rica, según el Women's Entrepreneurial Venture Scope (WEVentureScope, 2013), el país está clasificado en el 6o lugar de los 20 países analizados por su ambiente general para mujeres emprendedoras. Según dicho estudio, las mujeres empresarias tienen disponibles cursos de formación, asesoría administrativa y financiamiento. Unido a esto, la percepción de vulnerabilidad ante corrupción, soborno, delincuencia, robo y disturbios es relativamente baja. Estos últimos indicadores colocan al país en el 30\% superior del índice por tener los riesgos más bajos para la operación de un negocio por una mujer emprendedora. Además, menciona cierta infraestructura de servicios sociales de apoyo (i.e. cuidado de los niños), amplia disponibilidad de redes de negocio, así como programas de capacitación en habilidades básicas dirigidos a mujeres. Según dicho índice, la barrera más difícil de derribar para las mujeres en Costa Rica es el acceso al financiamiento, nuestro país se encuentra entre quienes más garantías exigen a las mujeres emprendedoras. Esto produce que las empresas dirigidas por mujeres reciban poco apoyo de los bancos para financiar inversiones.

Adicionalmente, es importante señalar algunas de las debilidades detectadas en el «Diagnóstico sobre la situación actual del Emprendedurismo en Centroamérica», (Lebendiker, Zevallos, Alonso y Petry, 2010), en lo concerniente al apoyo de las pymes:

- La institucionalidad existente no atiende en forma completa todas las etapas del proceso emprendedor.

- Inexistente coordinación de los esfuerzos para el fomento del emprendimiento, así como falta de un ente rector que articule estos esfuerzos.
- El marco regulatorio y legal no facilita la creación y desarrollo de nuevas empresas.

- Aunque el tema del emprendimiento está siendo abarcado en algunas universidades públicas, aún queda mucho por hacer para que el sector educativo se constituya en un actor importante dentro del proceso emprendedor del país.

- Insuficiencia en las opciones de financiamiento a los emprendedores. La mayoría de los esquemas de financiamiento se dirigen a empresas en operación, y no tanto al impulso requerido por nuevos proyectos productivos.

- Desde un enfoque de género, la participación de las mujeres en los proyectos emprendedores, se limita principalmente a actividades de subsistencia, limitándose de este modo su posibilidad de superación.

\section{METODOLOGÍA}

La metodología investigativa fue mixta: cuantitativa y cualitativa. Para lo cuantitativo se tomó como base los resultados del segundo diagnóstico nacional de Pymes, realizado por el Observatorio de Pymes de Costa Rica (Omipymes). Dicho trabajo aplicó una encuesta a empresas catalogadas como Pymes, formalmente establecidas dentro del territorio costarricense al 31 de enero de 2011, factibles de contactar mediante entrevista telefónica.

Es importante mencionar que la estructura de la encuesta aplicada por el Omipymes permite filtrar los resultados, logrando identificar las respuestas asociadas a empresas de mujeres. A partir de ello se pudo trabajar con la base de datos filtrada por género. Sin embargo, es importante considerar que la variable utilizada para filtrar la información tiene una limitación, considera empresas cuyo propietario al 
momento de la encuesta es una mujer, sin embargo, esto no siempre va a ser porque ella fundó la empresa, sino que puede deberse también a factores como la compra del negocio o la herencia del mismo.

En la Tabla 1 se resume la cantidad de encuestas clasificadas por el género del dueño actual, con la idea de tener una estimación del porcentaje de resultados obtenidos del total de encuestas. A pesar de que representan solamente un $16.98 \%$ del total, equivalente a 181 empresas, es una muestra aceptable para el presente estudio.

Tabla 1. Género del dueño actual del negocio Table 1. Current business owner's gender

\begin{tabular}{lcc}
\hline Propietario & Resultados & $\%$ \\
\hline Hombre & 887 & $83,02 \%$ \\
Mujer & 181 & $16,98 \%$ \\
Total & 1068 & $100,00 \%$
\end{tabular}

Fuente: elaboración propia a partir de la base de datos del Observatorio de Pymes (2011).

Considerando la muestra obtenida, es relevante aclarar aspectos relacionados con el nivel de confianza y margen de error. Los parámetros considerados para calcular el tamaño de muestra para el estudio del Observatorio son aplicables a la muestra global, es decir, sin distinción de género (comprendiendo tanto empresarias como empresarios). La estratificación de la muestra es por tamaño de empresay sector de actividad; por lo tanto, para esos desgloses se mantienen los parámetros establecidos. Dado lo anterior, para determinar la precisión de los resultados de una muestra de 181 mujeres emprendedoras costarricenses se aplica la siguiente fórmula:

$$
n_{o}=\frac{z_{\alpha / 2}^{2} p q}{d^{2}}=\frac{p q}{V}
$$

Obteniéndose que para una proporción $\mathrm{P}=$ 0,17 (estimada con base en la proporción de empresarias obtenida en el estudio del Observatorio), $Q=0,83$ (1- $P$ ), un nivel de confianza del 95\% (correspondiente a un valor $Z=1,96$ de la normal estándar) y un margen de error o diferencia máxima permisible de 5,5\% $(d=0,05)$, se obtiene un tamaño de muestra mínimo de $n=179$. Por tanto, este conjunto de 181 empresarias puede considerarse equivalente a una muestra con un nivel de confianza del $95 \%$ y un margen de error máximo de 5,5\%, valores que son bastante aceptables para un estudio en este ámbito.

En otras palabras, los resultados se pueden inferir a la población costarricense de mujeres propietarias de Pymes formalmente establecidas en las fechas del estudio con un nivel de confianza del $95 \%$ y un margen de error de $5.5 \%$.

Para profundizar en la metodología del Observatorio puede consultarse: http://omipymes.uned.ac.cr

Adicionalmente, para conocer el apoyo y obstáculos percibidos por las mujeres, así como los factores asociados al éxito, se realizó una serie de entrevistas a profundidad. Estas se efectuaron a representantes de entes que brindan apoyo a estas mujeres, así como a empresarias propiamente. La selección de la muestra fue por juicio de experto. Cabe destacar que estas entrevistas aportan mucho valor al estudio, por tanto, algunos de los aspectos planteados en los objetivos son más adecuados de obtener con informantes claves. Para tal efecto, se realizaron siete entrevistas semiestructuradas tanto en Costa Rica como México.

\section{RESULTADOS}

Este acápite muestra los resultados ordenados según los alcances planteados inicialmente: las motivaciones y características, así como el entorno, expresado en los obstáculos y apoyos percibidos. 


\section{Motivaciones}

Un primer resultado relevante fue determinar las motivaciones conducentes a las mujeres emprendedoras costarricenses a iniciar su propia empresa. En tal sentido, la Tabla 2 presenta los resultados.

Según los resultados de la Tabla 2, la principal motivación para iniciar un negocio fue independizarse $(65.51 \%$ de menciones), seguido del conocimiento del negocio, y en tercer lugar el autoempleo. Esto concuerda con los hallazgos de otros estudios (Lebendiker et al., 2012).

Buscando entender mejor, las entrevistas en profundidad dejan ver otras aristas del tema. Por ejemplo, las motivaciones se pueden relacionar también con muchas situaciones particulares de la mujer, dentro de las cuales está el crecimiento personal y profesional, así como en muchos casos sentirse útil, cumplir sus sueños, y salir adelante. En el caso concreto de las mujeres, se incluye también el tema de velar por el futuro de sus hijos.

Tabla 2. Motivaciones de las mujeres para iniciar un negocio en Costa Rica ( $n: 105)$ Table 2. Motivations of women to start a business in Costa Rica (n: 105)

\begin{tabular}{lcccc}
\hline & $S i$ & No & NS/NR & Total \\
\hline ¿Quería independizarse? & $65,51 \%$ & $33,59 \%$ & $0,91 \%$ & $100 \%$ \\
¿Conocía el negocio? & $53,35 \%$ & $45,75 \%$ & $0,91 \%$ & $100 \%$ \\
¿Autoempleo? & $52,92 \%$ & $43,47 \%$ & $3,61 \%$ & $100 \%$ \\
¿Viene de familia de empresarios? & $24,21 \%$ & $75,79 \%$ & $0,00 \%$ & $100 \%$ \\
¿Ingresos complementarios? & $16,64 \%$ & $83,36 \%$ & $0,00 \%$ & $100 \%$ \\
¿Hizo estudios de mercadeo? & $10,11 \%$ & $86,29 \%$ & $3,61 \%$ & $100 \%$ \\
¿Heredó el negocio? & $14,39 \%$ & $85,61 \%$ & $0,00 \%$ & $100 \%$ \\
¿Compró el negocio? & $12,12 \%$ & $86,97 \%$ & $0,91 \%$ & $100 \%$ \\
¿Otro? & $13,44 \%$ & $85,09 \%$ & $1,48 \%$ & $100 \%$ \\
\hline
\end{tabular}

Fuente: elaboración propia a partir de la base de datos del Observatorio de Pymes (2011).

Con las entrevistas se logró corroborar la predominancia de la necesidad de independizarse y las situaciones personales por las cuales estaba pasando en un momento dado la emprendedora. Por ejemplo, según entrevista realizada a la empresaria Yenory Cordero (comunicación personal, octubre 2013) vemos cómo en sus palabras se ratifica lo anterior, señalando «...bueno, después de un despido, decidíque lo mejor era tener mi propio negocio...». Adicionalmente, haciendo una comparación con los resultados obtenidos con mujeres empresarias mexicanas, se confirman las razones por las cuales las mujeres inician usualmente el negocio, un ejemplo es lo mencionado por Laura Caracheo (comunicación personal, octubre 2013),

Después de renunciar a mi trabajo, es ahí donde surge la pregunta: ¿y ahora a dónde dirijo mi vida? Y fue ahí donde tomé la decisión de iniciar este proyecto, en ese momento pasaba por una situación difícil de la adolescencia de tres hijos, estaba dentro de un matrimonio y por primera vez me encontré sin un salario fijo y también con la contrariedad de que el que fue mi esposo, (años después me divorcié), tenía planes para mí diferentes que eran apoyar en un negocio familiar y yo tomé la decisión de 
emprender mi propio instituto y no conté con su apoyo.

\section{Características y perfil}

En nuestro trabajo, otro de los temas de interés es el conjunto de características y perfiles presentes en las emprendedoras costarricenses y sus Pymes. Esto se relaciona en parte con la psicometría, se deben realizar pruebas psicológicas para tener un perfil exacto de características de las mujeres emprendedoras, sin embargo, también se puede formar un perfil básico a partir de algunas características halladas en la población encuestada.

El rango de edad más usual para iniciar los negocios fue entre 35 y 49 años (35.17\%), seguido por 25 y 34 años (26.65\%). En los rangos inferiores de edades parece ser que, aunque las mujeres sí emprenden negocios, lo hacen en una proporción menor.

Por otro lado, si se analizan los rangos de edades actuales, un $37.25 \%$ de las emprendedoras posee más de 50 años, seguido de un $35.71 \%$ de mujeres entre los 35 a 49 años, lo cual coincide con las respuestas de la pregunta anterior, es decir, las empresas establecidas están lideradas principalmente por mujeres no menores de 34 años.

En relación con la escolaridad, al iniciar el negocio, se determina que un $58.61 \%$ de ellas tenían nivel universitario y un $15.93 \%$ nivel de secundaria. Un dato interesante: únicamente un $3.57 \%$ tenía un posgrado universitario. Sin embargo, analizando el nivel de escolaridad al momento de la encuesta, se aprecian porcentajes de posgrado mayor y por lo tanto disminución de los otros niveles. No obstante, dicho aumento en mujeres con posgrados no cambia la situación general, la mayor proporción de emprendedoras posee solo formación universitaria de grado y secundaria
(64.19\% y $15.68 \%$ respectivamente). Esto indudablemente se relaciona con las características de las empresas analizadas (son formales), así como la cobertura geográfica del estudio, pues otros autores han mostrado resultados diferentes en la composición y características de las empresarias (BermúdezCarrillo, 2014; Brenes y Bermúdez, 2013).

En cuanto a las empresas como tales, el 53.04\% pertenece al sector servicios, seguidas por un 30.39\% de comercio.

En el tema exportación, hay poco desarrollo. Un $92.27 \%$ no había realizado ninguna en los doce meses previos a la encuesta. Solamente un $7.20 \%$ manifestó que su servicio o producto es exportado por otra empresa y un $4.49 \%$ indicó que es insumo para una empresa exportadora. Asimismo, se determinó que, entre las empresas exportadoras de mujeres, el principal producto es de naturaleza agrícola (20.62\%), seguido por software (12.75\%), alimentos (11.93\%), textiles (10.48\%), entre los más relevantes.

Otro aspecto interesante es determinar la participación general de las mujeres emprendedoras costarricenses en las Pymes. Al respecto, se pudo identificar que de las empresas encuestadas por el Observatorio un 16.98\% correspondían a empresas propiedad de una mujer $y$ en contraste un $83.02 \%$ a hombres.

Estos resultados difieren en proporción, más no en la dirección con los de Lebendiker, et al. (2012): los hombres emprenden más que las mujeres. Según estos autores, en el caso de Costa Rica, la incidencia por sexo en las actividades emprendedoras no difiere mucho entre hombres y mujeres, en donde el $13.2 \%$ de los emprendedores son hombres quienes manifiestan ser emprendedores en etapas iniciales, versus el $13.7 \%$ de mujeres. En el caso 
de los negocios establecidos, el 6.5\% son de hombres y el $3.1 \%$ de mujeres.

Respecto al lugar en donde se ubica la cantidad más representativa de las Pymes lideradas por mujeres emprendedoras, se evidencia una importante concentración en las provincias de San José (47.40\%), Alajuela (19.79\%) y Heredia (12.60\%), en contraste con las otras provincias, en las cuales se distribuyen el restante $20.21 \%$. Acorde con lo mencionado por la señora Kattya Rodríguez (comunicación personal, octubre 2013), estos resultados no deben interpretarse como que las mujeres en las otras provincias no emprendan o no tengan negocios. Lo que sucede es que no son calificados por su naturaleza como empresas, pues no cumplen con las características mínimas para ello desde el punto de vista legal (Bermúdez-Carrillo, 2014).

\section{Obstáculos y apoyos}

Dentro de los principales obstáculos para iniciar un negocio se encuentra el acceso a los recursos, por lo cual en la Tabla 3 se describe la fuente de donde fueron obtenidos, en el caso de las mujeres bajo estudio.

Se puede observar cómo un porcentaje muy representativo (79.01\%) de las mujeres encuestadas iniciaron su negocio con fondos propios, seguido de prestamistas informales, bancos públicos y préstamos empresariales.

Tabla 3. Fondos utilizados por emprendedoras costarricenses para iniciar un negocio ( $n: 143$ )

Table 3. Funds used by Costa Rican entrepreneurs to start a business ( $\mathrm{n}: 143$ )

\begin{tabular}{l|llll}
\hline & Si & No & NS/NR & Total \\
\hline Recursos propios & $79,01 \%$ & $16,57 \%$ & $4,42 \%$ & $100,00 \%$ \\
Prestamistas informales & $44,12 \%$ & $44,12 \%$ & $11,76 \%$ & $100,00 \%$ \\
Bancos públicos & $42,11 \%$ & $36,84 \%$ & $21,05 \%$ & $100,00 \%$ \\
Préstamos empresariales & $18,42 \%$ & $60,53 \%$ & $21,05 \%$ & $100,00 \%$ \\
Organización de microfinanzas & $13,51 \%$ & $62,16 \%$ & $24,32 \%$ & $100,00 \%$ \\
Tarjetas de crédito & $2,63 \%$ & $76,32 \%$ & $21,05 \%$ & $100,00 \%$ \\
Cooperativa & $2,63 \%$ & $76,32 \%$ & $21,05 \%$ & $100,00 \%$ \\
Parientes o amigos & $2,63 \%$ & $76,32 \%$ & $21,05 \%$ & $100,00 \%$ \\
Fondos PROPYME & $2,63 \%$ & $76,32 \%$ & $21,05 \%$ & $100,00 \%$ \\
Bancos privados & $2,63 \%$ & $76,32 \%$ & $21,05 \%$ & $100,00 \%$ \\
Otros & $0,00 \%$ & $78,95 \%$ & $21,05 \%$ & $100,00 \%$ \\
Proveedores & $0,00 \%$ & $78,95 \%$ & $21,05 \%$ & $100,00 \%$ \\
Emisión de acciones & $0,00 \%$ & $78,95 \%$ & $21,05 \%$ & $100,00 \%$ \\
\hline
\end{tabular}

Fuente: elaboración propia a partir de la base de datos del Observatorio de Pymes (2011). 
En general, las entrevistas a profundidad realizadas arrojaron respuestas muy similares. Uno de los principales obstáculos fueron los recursos para iniciar la empresa. En algunos casos provinieron de algún familiar cercano, como un hermano o el esposo, porque era difícil conseguir un crédito. Según lo indicado por las entrevistadas, en ocasiones se tramitó un crédito bancario, sin embargo, se complicó el acceso al ser un negocio incipiente. Algunas veces se realizó por medio de financiamiento enfocado a Pymes en general, pero no a mujeres en particular.

Muestra de lo anterior es lo señalado por Gabriela Chaverri, propietaria de la empresa Color Concepts "Mi condición de mujer fue determinante, en ese entonces costaba más, tal vez el banquero dudaba más en si le daba un préstamo a una mujer» (comunicación personal, octubre 2013).

Otro de los aspectos calificados como obstáculos para iniciar un negocio es la realización de trámites, en tal sentido la Tabla 4 muestra los resultados ligados a dicho tema.

De acuerdo con los resultados del cuadro anterior, se puede inferir que excluyendo las respuestas de «ninguno», «no responde» o «no sabe», los trámites con mayor dificultad son los del Ministerio de Salud (13.80\%), municipalidades (11.97\%) y patentes (9.04\%).

Aunque los resultados no muestran cifras contundentes es interesante lo señalado en diferentes ámbitos, sobre la existencia de consenso en que los trámites en Costa Rica son uno de los temas prioritarios por resolver en aras de aumentar la competitividad (Otárola, 2015).

Asimismo, según fue señalado previamente, la Organización de las Naciones Unidas (2010) ha expresado que las mujeres de la región enfrentan barreras mucho más complejas para su participación en la economía formal versus sus contrapartes en países desarrollados: escasas políticas y programas de estímulo, exceso de normativas procedimental, falta acceso al crédito. Las principales dificultades son la falta de experiencia empresarial, insuficiente formación específica (administración/marketing), barreras en acceso a fuentes de crédito y financiamiento, obstáculos para acceso a redes de comercialización, excesivos trámites y procedimientos para iniciar sus negocios.

Tabla 4. Trámites de mayor dificultad para las mujeres al iniciar una empresa en Costa Rica ( $\mathrm{n}: 171)$

Table 4. Procedures of greater difficulty for women when starting a company in Costa Rica (n:171)

\begin{tabular}{l|cc}
\hline & SI & $\%$ \\
\hline Ninguno & 31 & $18,43 \%$ \\
No responde & 27 & $16,00 \%$ \\
Ministerio de Salud & 24 & $13,80 \%$ \\
No lo sabe & 23 & $13,36 \%$ \\
Municipalidad & 20 & $11,97 \%$ \\
Patentes & 15 & $9,04 \%$ \\
Otros & 9 & $5,10 \%$ \\
Aprobación de documentos general & 5 & $2,90 \%$ \\
Todos & 4 & $2,58 \%$ \\
Otras instituciones públicas & 4 & $2,11 \%$ \\
Ya existían & 3 & $1,66 \%$ \\
Ministro Hacienda & 2 & $1,31 \%$ \\
CCss & 2 & $0,98 \%$ \\
Certificaciones & 1 & $0,75 \%$ \\
\hline
\end{tabular}

Fuente: elaboración propia a partir de la base de datos del Observatorio de Pymes (2011).

Otro tema relevante es si la empresa ha utilizado la ayuda de los programas de apoyo a las pymes, para lo cual se indagó por parte del Observatorio si esto fue así, y eventualmente el nombre del programa y los beneficios recibidos. 
Al respecto, se determinó que del total de empresas encuestadas únicamente un 10.15\% utilizaron la ayuda de programas a Pymes, dentro de los cuales figuran entre los primeros lugares los del Banco Nacional de Costa Rica y del Ministerio de Economía, Industria y Comercio de Costa Rica. Los principales beneficios fueron de recurso humano 0 capacitación y en último lugar el apoyo a la producción.

Al respecto, es interesante destacar cómo mediante las entrevistas realizadas se halló que según Cordero (comunicación personal, octubre 2013) una de sus necesidades es mejorar sus técnicas de producción y esa es una de las deficiencias presentes en los programas de apoyo precisamente. Asimismo, según Lilly Cheng (comunicación personal, octubre 2013), quien es coordinadora del programa de desarrollo gerencial de la Dirección de Extensión de la Universidad Estatal a Distancia de Costa Rica, un tema trascendental en cuanto a emprendedoras es que ellas usualmente solo saben producir o vender, lo cual en ocasiones dificulta el desarrollo de sus negocios.

En cuanto al apoyo financiero, de educación y formación, Kattya Rodríguez (comunicación personal, octubre 2013), quien es directora de Banca Mujer del Banco Nacional de Costa Rica, menciona que dicho programa, entre otros aspectos, prepara a las mujeres para poder presentar un proyecto viable al banco, al cual denomina un plan a la medida. Lo que se busca con ello es materializar la idea de negocio, a la vez que se trabaja en el empoderamiento de las empresarias. En este sentido, el Banco trata de generar ciertas características diferenciadoras de estos programas para enfocarlos en mujeres.

Además, los programas son pre y post crédito, pues posterior al otorgamiento del financiamiento se dan programas de actualización. Sin embargo, ella recalca la necesidad de mejorar los fondos asignados por el Gobierno para este tipo de capacitaciones, mejorar la metodología docente y adecuar los programas a las necesidades de mujeres, por ejemplo, en cuestiones de horarios.

\section{CONCLUSIONES}

El objetivo del presente artículo fue analizar cómo se caracterizan las emprendedoras costarricenses propietarias de pequeñas y medianas empresas, considerando aspectos específicos, como las motivaciones para iniciar su negocio, características personales y de sus empresas, así como principales obstáculos y apoyos percibidos por ellas.

En cuanto a las motivaciones, predominan las de carácter personal ligadas al deseo de independencia y conocimiento del negocio. Están más enfocadas al aprovechamiento de oportunidades versus necesidad de subsistencia. Así mismo, su rol familiar es un motivador adicional, por ejemplo, velar por el futuro de sus hijos.

En cuanto a las características, se presentó un perfil de la mujer emprendedora donde destaca su formación universitaria, rango de edad actual y al formar su empresa. Esto puede dar pie para una mejor identificación de sus necesidades y requerimientos para diseñar programas de apoyo.

Lo anterior es importante por cuanto otra conclusión del estudio es que las mujeres perciben obstáculos importantes en su proceso emprendedor y los programas de apoyo no están ayudando a solventarlos. Temas como el mejoramiento de la producción y el acceso al financiamiento parecieran ser materia pendiente en Costa Rica.

De las conclusiones emanan algunas recomendaciones para los tomadores de 
decisión en la materia, así como futuras líneas de acción. Por ejemplo, en el tema de las motivaciones femeninas para emprender en Costa Rica, sería muy interesante considerar empresas del sector formal e informal, pues existen realidades muy diferentes entre ellas. Por ende, las necesidades para fomentar el proceso emprendedor y su éxito pueden variar entre ambos sectores.

Una oportunidad de mejora es agilizar los trámites para la formalización y acompañamiento inicial de las empresas. Además, se podrían implementar programas educativos desde los colegios y universidades con miras al empoderamiento y desarrollo de características personales necesarias para el espíritu emprendedor: toma de decisiones, autoconfianza y persistencia.

Es importante, también, agilizar los requisitos y procesos para acceder los programas de apoyo a las emprendedoras costarricenses, aumentar aquellos enfocados en procesos y técnicas de producción, así como mejorar los programas actuales de seguimiento y acompañamiento, enfocándolos a las necesidades femeninas, por ejemplo, considerando la flexibilidad de horarios y otros temas particulares de las mujeres por los roles sociales que desempeñan.

Por otro lado, se considera importante generar bases de datos con información por género del propietario del negocio. Ello tanto en entidades financieras como en programas de apoyo. Así se podrá generar más conocimiento sobre los emprendimientos femeninos y sus características, logrando con ello tener mejor conocimiento sobre cuáles son las necesidades latentes.

Nuestro artículo tiene limitaciones como todo trabajo científico de esta índole. La principal es la disponibilidad de información, pues se trabajó con una base de datos no hecha ad hoc.
Además, el no poder disponer de información sobre empresas no formales, como fue señalado previamente, llama a mirar los resultados con mesura y reconociendo que su aplicabilidad es para empresas formales establecidas al momento del estudio.

\section{REFERENCIAS}

Brenes, L. y Bermúdez, L. (2013). Diferencias por género en el emprendimiento empresarial costarricense. Tec Empresarial, 7(2), 19-27.

Bermúdez-Carrillo, L. A. (2014). Características de las Pymes de Guanacaste. InterSedes. XV(30), 6-21.

GEM (2007). Report on Women and Entrepreneurship. Global Entrepreneurship Monitor.www.gemconsortium.org

GEM (2012). Women's Report. Global Entrepreneurship

Monitor.www.gemconsortium.org

Gilder, G. (1984). Empresarialidad: contribución al crecimiento y al bienestar económico. México: Mc Graw-Hill.

Kantis H., Angelelli P. y Koening, M. (2004). Desarrollo emprendedor: América Latina y la experiencia internacional (Quinta ed.). Washington, DC, USA: Interamerican Development Bank.

Lebendiker, M.; Zevallos, E.; Alonso, E. y Petry, P. (2010). Diagnóstico sobre la situación actual del emprendedurismo en Centroamérica. Asociación Incubadora Parque Tec. Disponible en: http://www.parquetec.org/publicaciones. php 
Lebendiker M.; Petry P.; Herrera R. y Velásquez G. (2012). Reporte Nacional GEM 2012: Ia situación del emprendimiento en Costa Rica. Costa Rica: Editorama, S.A.

Ministerio de Economía Industria y Comercio MEIC- (2010). Política Nacional de Emprendimiento: Costa Rica Emprende.

McClelland, D. C. (1961). The Achieving Society. Princeton, N. J.: D. Van. Nostrand Co.

Olmos, J. y Arrayales, J. (2007). Tu potencial emprendedor. México: Pearson Educación.

Organización de las Naciones Unidas (2010). Mujeres emprendedoras en América Latina y el Caribe: realidades, obstáculos y desafíos. Santiago de Chile. Disponible en: http://www.cepal.org/es/publicaciones/58 18-mujeres-emprendedoras-en-americalatina-y-el-caribe-realidades-obstaculos-y

Otárola, S. (2015). Sector público y privado definen temas de interés común en Consejo de Competitividad. Disponible en: http://gobierno.cr/sector-publico-yprivado-definen-temas-de-interes-comunen-consejo-de-competitividad/

WEVentureScope (2013). Índice del entorno empresarial para emprendedoras. Retrieved octubre 24, 2013, from Índice del entorno empresarial para emprendedoras: http://www.weventurescope.com/es/Hom e/Ranking 